Article

\title{
Investigation and Validation of the Time-Varying Characteristic for the GPS Differential Code Bias
}

\author{
Haojun $\mathrm{Li}^{1}$, Jingxin $\mathrm{Xiao}^{1}$ and Weidong $\mathrm{Zhu}^{2, *}$ \\ 1 College of Surveying and Geo-Informatics, Tongji University, Shanghai 200092, China; \\ lhjch@tongji.edu.cn (H.L.); 1633286@tongji.edu.cn (J.X.) \\ 2 College of Marine Sciences, Shanghai Ocean University, Shanghai 201306, China \\ * Correspondence: wdzhu@shou.edu.cn
}

Received: 12 January 2019; Accepted: 16 February 2019; Published: 19 February 2019

\begin{abstract}
The time-varying characteristic of the bias in the GPS code observation is investigated using triple-frequency observations. The method for estimating the combined code bias is presented and the twelve-month (1 January-31 December 2016) triple-frequency GPS data set from 114 International GNSS Service (IGS) stations is processed to analyze the characteristic of the combined code bias. The results show that the main periods of the combined code bias are 12, 8, 6, 4, 4.8 and $2.67 \mathrm{~h}$. The time-varying characteristic of the combined code bias, which is the combination of differential code bias (DCB) (P1-P5) and DCB (P1-P2), shows that the real satellite DCBs are also time-varying. The difference between the two sets of the computed constant parts of the combined code bias, with the IGS DCB products of DCB (P1-P2) and DCB (P1-P2) and the mean of the estimated 24-h combined code bias series, further show that the combined code bias cannot be replaced by the DCB (P1-P2) and DCB (P1-P5) products. The time-varying part of inter-frequency clock bias (IFCB) can be estimated by the phase and code observations and the phase based IFCB is the combinations of the triple-frequency satellite uncalibrated phase delays (UPDs) and the code-based IFCB is the function of the DCBs. The performances of the computed the IFCB with different methods in single point positioning indicate that the accuracy for the constant part of the combined code bias is reduced, when the IGS DCB products are used to compute. These performances also show that the time-varying part of IFCB estimated with phase observation is better than that of code observation. The predicted results show that $98 \%$ of the predicted constant part of the combined code bias can be corrected and the attenuation of the predicted accuracy is much less evident. However, the accuracy of the predicted time-varying part decreases significantly with the predicted time.
\end{abstract}

Keywords: hardware delay bias; uncalibrated phase delay; GPS satellite clock; precise point positioning; inter-frequency clock bias

\section{Introduction}

There are biases between Global navigation satellite system (GNSS) different observations due to effects of space environment and frequency-dependent signal hardware delays at the satellite and the receiver. The GNSS biases are generally analyzed and investigated based on the redundant observations and the geometry-free observations. They can be classified into two types of code and phase observations and have obtained much attention from many authors and this area of research has deeply developed in recent years [1]. The differential code bias (DCB) is one of the biases referring to codes modulated on difference carriers or on the same carrier. The DCB (P1-P2) is the most common bias between the P1 and P2 observations [2-4]. The DCB (P1-P2) includes receiver and satellite parts and is generally estimated together with the ionosphere model parameters using GNSS code geometry-free combination from ground networks stations $[5,6]$. Its accuracy is easily affected by 
the pre-set ionosphere model (e.g., polynomials and spherical harmonics) errors and the noise of the code observations. The ionosphere model parameters are also estimated using the raw observations based precise point positioning (PPP) [7]. In this method, the phase and code observations are used to estimate the ionosphere model parameters, but the receiver DCB (P1-P2) was just computed and the satellite DCB (P1-P2) was corrected using International GNSS Service (IGS) [8] products. Most of the GNSS biases, for example the DCB (P1-P2), DCB (C1-P1) [9] and the inter-system bias (ISB) of phase observation [10] are considered and computed as constant values and a daily or weekly constant is provided. Some of the time-varying biases are noticed as the cognition of GNSS and its observations deepen in recent years. The time-varying uncalibrated phase delay (UPD) of narrow lane is analyzed so that it is computed with a step-size of $15 \mathrm{~min}$ [11]. The carrier-phase inter-frequency biases (IFB) of GLONASS receivers are computed and their variation is analyzed [12,13], the short-term temporal variations of receiver differential phase biases are estimated, and their characteristics are discussed. The time-varying triple-frequency GPS satellite inter-frequency clock bias (IFCB) is investigated [14-16] using the triple-frequency GPS observations. Then the GPS biases, which have the periodic and time-varying characteristic, are gradually noted and analyzed.

The biases, especially the time-varying bias, in the code observation not only affect the accuracy of the code observation but also the high-quality float ambiguity solution. This requires rigorous modeling of all error sources affecting carrier phase and code observations in PPP data processing, and the corresponding bias should be precisely calibrated for code-based positioning [17-19], timing [20], and ionosphere modeling [21-23]. Currently, the code biases, for example the DCB (P1-P2) of the satellites and receivers, are estimated as constant values for each day and have been routinely published by individual Ionospheric Associate Analysis Center (IAAC, including CODE, JPL, UPC, and ESA) of the IGS since 1998 [24]. Zhong et al. [25] analyzed the long-term variation of the estimated DCB and drew the conclusion that this variation is mainly associated with GPS satellite replacement using the DCB products. Choi and Lee [26] discussed the influence of grounding on GPS receiver differential code biases and Yasyukevich et al. [27] shows the influence of GPS/GLONASS differential code biases on the determination accuracy of the absolute total electron content in the ionosphere. However, the time-varying characteristic of the bias in the code observation is not covered by the current studies and products. In order to further improve the accuracy of the code-based positioning, the time-varying characteristic of the code bias is investigated. Next the application of the code bias in single point positioning (SPP) is implemented to analyze the time-varying characteristic of the code bias. Finally, the research findings and outlooks are summarized.

\section{Biases in the Code and Phase Observations}

The biases in the raw, ionosphere-free and differenced ionosphere-free code and phase observations are discussed and their characteristic is analyzed. Meanwhile, the strategy for estimating the combined code biases is presented. Application of the estimated time-varying combined code bias in the positioning is discussed.

\subsection{Biases in the Raw Code and Phase Combinations}

For a triple-frequency user, the raw phase and code observations are written as:

$$
\begin{aligned}
& L_{1}=\rho+N_{r}^{1} \lambda_{1}+U P D_{r}^{1}-U P D_{1}^{s}+\delta_{r}-\delta^{s}+I_{r}^{s}-T_{r}^{s}+\varepsilon_{1} \\
& P_{1}=\rho+b_{r}^{1}-b_{1}^{s}+\delta_{r}-\delta^{s}-I_{r}^{s}-T_{r}^{s}+\omega_{1} \\
& L_{2}=\rho+N_{r}^{2} \lambda_{2}+U P D_{r}^{2}-U P D_{2}^{s}+\delta_{r}-\delta^{s}+\frac{f_{1}^{2}}{f_{2}^{2}} I_{r}^{s}-T_{r}^{s}+\varepsilon_{2} \\
& P_{2}=\rho+b_{r}^{2}-b_{2}^{s}+\delta_{r}-\delta^{s}-\frac{f_{1}^{2}}{f_{2}^{2}} I_{r}^{s}-T_{r}^{s}+\omega_{2} \\
& L_{5}=\rho+N_{r}^{5} \lambda_{5}+U P D_{r}^{5}-U P D_{5}^{s}+\delta_{r}-\delta^{s}+\frac{f_{1}^{2}}{f_{5}^{2}} I_{r}^{s}-T_{r}^{s}+\varepsilon_{5} \\
& P_{5}=\rho+b_{r}^{5}-b_{5}^{s}+\delta_{r}-\delta^{s}-\frac{f_{1}^{2}}{f_{5}^{2}} I_{r}^{s}-T_{r}^{s}+\omega_{5}
\end{aligned}
$$


where $\rho$ is the satellite-to-receiver range, $T_{r}^{s}$ is tropospheric delay, $I_{r}^{s}$ is the slant ionospheric delay of L1 observation, $f_{i}(i=1,2,5)$ are the carrier frequencies of L1, L2 and L5, $\delta_{r}$ and $\delta^{s}$ are the receiver and satellite clock errors in meter, $b_{i}^{s}(i=1,2,5)$, which contains the constant and time-varying parts, are the satellite hardware delay biases of P1, P2 and P5 observations, $b_{r}^{i}(i=1,2,5)$ are the receiver hardware delay biases of P1, P2 and P5 observations, $\varepsilon_{i}(i=1,2,5)$ are noises of L1, L2 and L5 observations, $\omega_{i}(i=1,2,5)$ are noises of P1, P2 and P5 observations, $N_{r}^{i}(i=1,2,5)$ are the ambiguities of L1, L2 and L5 observations, $\lambda_{i}(i=1,2,5)$ are the wavelengths of L1, L2 and L5 observations, $\operatorname{UPD}_{r}^{i}(i=1,2,5)$ are the receiver UPD of L1, L2 and L5 observations, $U P D_{i}^{s}(i=1,2,5)$, which contains the constant and time-varying parts, are the satellite UPDs of L1, L2 and L5 observations. It is difficult to compute the satellite or receiver hardware delay biases in the raw code observations and the UPDs in the raw phase observations using their corresponding observations.

\subsection{Combined Biases in the Code and Phase Combinations}

The code geometry-free combination is generally used to compute the ionosphere model parameters and DCB, which refers to the reference observation. The time-varying part of DCB is absorbed by the ionosphere model parameters so that the DCB (P1-P2) and DCB (P1-P5) products published by IGS are just the constant part. The UPD in the raw phase observation is correlative with the phase ambiguity and the fractional cycle part is generally computed based on PPP ambiguity results. However, the PPP ambiguity results are affected by the used products of PPP and the characteristic of the UPD is dominated by the used products and observations of the PPP user side. It is a complex procedure and issue.

Two groups of the ionosphere-free carrier phase and code observations for the triple-frequency GPS are formed as:

$$
\begin{array}{ll}
\operatorname{IF}\left(L_{1}, L_{2}\right)=\rho & +\left(a_{1} N_{r}^{1} \lambda_{1}-a_{2} N_{r}^{2} \lambda_{2}\right)+\left(a_{1} U P D_{r}^{1}-a_{2} U P D_{r}^{2}\right) \\
& -\left(a_{1} U P D_{1}^{s}-a_{2} U P D_{2}^{s}\right)+\delta_{r}-\delta^{s}-T_{r}^{s}+\varepsilon_{1,2} \\
\operatorname{IF}\left(P_{1}, P_{2}\right)=\rho & +\left(a_{1} b_{r}^{1}-a_{2} b_{r}^{2}\right)-\left(a_{1} b_{1}^{s}-a_{2} b_{2}^{s}\right)+\delta_{r}-\delta^{s}-T_{r}^{s}+\omega_{1,2} \\
\operatorname{IF}\left(L_{1}, L_{5}\right)=\rho & +\left(a_{3} N_{r}^{1} \lambda_{1}-a_{4} N_{r}^{5} \lambda_{5}\right)+\left(a_{3} U P D_{r}^{1}-a_{4} U P D_{r}^{5}\right) \\
& -\left(a_{3} U P D_{1}^{s}-a_{4} U P D_{5}^{s}\right)+\delta_{r}-\delta^{s}-T_{r}^{s}+\varepsilon_{1,5} \\
\operatorname{IF}\left(P_{1}, P_{5}\right)=\rho & +\left(a_{3} b_{r}^{1}-a_{4} b_{r}^{5}\right)-\left(a_{3} b_{1}^{s}-a_{4} b_{5}^{s}\right)+\delta_{r}-\delta^{s}-T_{r}^{s}+\omega_{1,5}
\end{array}
$$

where $a_{1}=f_{1}^{2} /\left(f_{1}^{2}-f_{2}^{2}\right), a_{2}=f_{2}^{2} /\left(f_{1}^{2}-f_{2}^{2}\right), a_{3}=f_{1}^{2} /\left(f_{1}^{2}-f_{5}^{2}\right)$ and $a_{4}=f_{5}^{2} /\left(f_{1}^{2}-f_{5}^{2}\right), \varepsilon_{1,2}$ and $\varepsilon_{1,5}$ are noises of the ionosphere-free carrier observations L1/L2 and L1/L5, $\omega_{1,2}$ and $\omega_{1,5}$ are noises of the ionosphere-free carrier and code observations of P1/P2 and P1/P5. The satellite biases in the P1/P2 and P1/P5 are:

$$
\begin{aligned}
& b_{1,2}^{s}=b_{1}^{s}+a_{2} \operatorname{DCB}^{s}\left(P_{1}-P_{2}\right)=b_{2}^{s}+a_{1} \operatorname{DCB}^{s}\left(P_{1}-P_{2}\right) \\
& b_{1,5}^{s}=b_{1}^{s}+a_{4} \operatorname{DCB}^{s}\left(P_{1}-P_{5}\right)=b_{5}^{s}+a_{1} \operatorname{DCB}^{s}\left(P_{1}-P_{5}\right)
\end{aligned}
$$

The satellite biases in the L1/L2 and L1/L5 are:

$$
\begin{aligned}
& \mathrm{UPD}_{1,2}^{s}=\mathrm{UPD}_{1}^{s}+a_{2}\left(\mathrm{UPD}_{1}^{s}-\mathrm{UPD}_{2}^{s}\right)=\mathrm{UPD}_{2}^{s}+a_{1}\left(\mathrm{UPD}_{1}^{s}-\mathrm{UPD}_{2}^{s}\right) \\
& \mathrm{UPD}_{1,5}^{s}=\mathrm{UPD}_{1}^{s}+a_{4}\left(\mathrm{UPD}_{1}^{s}-\mathrm{UPD}_{5}^{s}\right)=\mathrm{UPD}_{5}^{s}+a_{3}\left(\mathrm{UPD}_{1}^{s}-\mathrm{UPD}_{5}^{s}\right)
\end{aligned}
$$

Equations (3) and (4) show that the biases in the ionosphere-free carrier and code observations are the combined bias. Similar to the biases in the raw phase and code observations, it is impossible to compute the biases of $b_{1,2}^{s}, b_{1,5}^{s}, \mathrm{UPD}_{1,2}^{s}$ and $\mathrm{UPD}_{1,5}^{s}$ using Equation (2). These satellite biases 
are absorbed by the estimated, reparameterized satellite clock errors in satellite clock estimating. The differenced ionosphere-free phase [14] and code observations [28] are:

$$
\begin{aligned}
& \operatorname{DIF}\left(L_{1}, L_{2}, L_{5}\right)=\operatorname{IF}\left(L_{1}, L_{5}\right)-\operatorname{IF}\left(L_{1}, L_{2}\right) \\
& =\left(a_{3} N_{r}^{1} \lambda_{1}-a_{4} N_{r}^{5} \lambda_{5}\right)+\left(a_{3} U P D_{r}^{1}-a_{4} U P D_{r}^{5}\right)-\left(a_{3} U P D_{1}^{s}-a_{4} U P D_{5}^{s}\right) \\
& -\left(a_{1} N_{r}^{1} \lambda_{1}-a_{2} N_{r}^{2} \lambda_{2}\right)-\left(a_{1} U P D_{r}^{1}-a_{2} U P D_{r}^{2}\right)+\left(a_{1} U P D_{1}^{s}-a_{2} U P D_{2}^{s}\right)+\varepsilon_{1,5}-\varepsilon_{1,2} \\
& \operatorname{DIF}\left(P_{1}, P_{2}, P_{5}\right)=I F\left(P_{1}, P_{5}\right)-I F\left(P_{1}, P_{2}\right) \\
& =\left(a_{3} b_{r}^{1}-a_{4} b_{r}^{5}\right)-\left(a_{3} b_{1}^{s}-a_{4} b_{5}^{s}\right)-\left(a_{1} b_{r}^{1}-a_{2} b_{r}^{2}\right)+\left(a_{1} b_{1}^{s}-a_{2} b_{2}^{s}\right)+\omega_{1,5}-\omega_{1,2} \\
& =a_{2} \mathrm{DCB}^{s}\left(P_{1}-P_{2}\right)-a_{4} \mathrm{DCB}^{s}\left(P_{1}-P_{5}\right)+a_{4} \mathrm{DCB}_{r}\left(P_{1}-P_{5}\right)-a_{2} \mathrm{DCB}_{r}\left(P_{1}-P_{2}\right) \\
& +\omega_{1,5}-\omega_{1,2}
\end{aligned}
$$

Equation (5) shows that the differenced ionosphere-free phase observation is the expression of the ambiguity and combined UPDs and the differenced ionosphere-free code observation is the combined hardware delay biases except the observation noise. The satellite combined biases in the differenced ionosphere-free code and phase observations are:

$$
\begin{aligned}
& b_{1,2,5}^{s}=a_{4} \mathrm{DCB}^{\mathcal{S}}\left(P_{1}-P_{5}\right)-a_{2} \mathrm{DCB}^{\mathcal{S}}\left(P_{1}-P_{2}\right) \\
& \mathrm{UPD}_{1,2,5}^{s}=a_{4}\left(\mathrm{UPD}_{1}^{s}-\mathrm{UPD}_{5}^{s}\right)-a_{2}\left(\mathrm{UPD}_{1}^{s}-\mathrm{UPD}_{2}^{s}\right)
\end{aligned}
$$

The defined IFCB is estimated with Equation (5). In IFCB computing, the constant part uses the differenced ionosphere-free code observation and the time-varying part uses the differenced ionosphere-free phase observation. The estimation method neglects the time-varying characteristic of the bias in the GPS code observation.

\subsection{Estimation of the Combined Code Bias}

Equation (5) indicates that it is difficult to separate the receiver or satellite combined code bias from their combination. Generally, the satellite-differenced observation is used based on the selected reference satellite. However, the rising and setting of the selected reference satellite results in the complexity of processing. The reference is also determined:

$$
\sum_{j=1}^{d}\left(b_{1,2,5}^{r}\right)_{j}=0
$$

where $\left(b_{1,2,5}^{s}\right)_{j}$ is the combined code bias at observation station $j ; d$ is the number of the observation station. When this reference is introduced, the parameterization equation of the combined code bias is expressed as:

$$
\left[\begin{array}{cc}
e_{1 \times d} & 0_{1 \times t} \\
I_{d \times d} \otimes e_{t \times 1} & e_{d \times 1} \otimes I_{t \times t}
\end{array}\right]\left[\begin{array}{c}
\left(b_{1,2,5}^{r}\right)_{1} \\
\ldots \\
\left(b_{1,2,5}^{r}\right)_{d} \\
\left(b_{1,2,5}^{s}\right)_{1} \\
\ldots \\
\left(b_{1,2,5}^{s}\right)_{t}
\end{array}\right]=\left[\begin{array}{c}
0 \\
\left(\operatorname{DIF}\left(P_{1}, P_{2}, P_{5}\right)\right)_{1,1} \\
\ldots \\
\left(D I F\left(P_{1}, P_{2}, P_{5}\right)\right)_{1, t} \\
\ldots \\
\left(D I F\left(P_{1}, P_{2}, P_{5}\right)\right)_{d, t}
\end{array}\right]
$$

where $I_{d \times d}$ and $I_{t \times t}$ are identity matrix, $e_{1 \times d}$ is a vector with each element is $1, t$ is the satellite number. The estimator of least-square adjustment is implemented to compute the epoch-wise combined code bias. In processing, the elevation-dependent weight is used:

$$
w_{m}=\left\{\begin{array}{cc}
1 & 30^{0} \leq \theta_{m} \leq 90^{0} \\
2 \sin \left(\theta_{m}\right) & 10^{0} \leq \theta_{m}<30^{0}
\end{array}\right\}
$$


where $\theta_{m}$ is the satellite elevation at $m$ epoch. The references of the estimated combined code biases for each satellite are consistent and it can be absorbed by the receiver clock in positioning so that the positioning result is not affected. The combined biases in the differenced ionosphere-free code observations differ from that of the raw and the ionosphere-free code observations and they can be computed using the corresponding observations. This is meaningful for studying the characteristic of the biases in GPS observation, especially that of code observation.

\subsection{Validation of the Time-Varying Code Bias}

The satellite-dependent biases are absorbed by the estimated, reparameterized satellite clock error in the satellite clock estimating [19]. Therefore, the code bias is very important for computing the reparameterized satellite clock of the corresponding observation. The combined bias for the differenced ionosphere-free combinations is named as IFCB and used to compute the reparameterized satellite clock errors of L1/L5 and P1/P5:

$$
\delta_{1,5}^{s}=\delta_{1,2}^{s}+I F C B^{s}
$$

where $\delta_{1,5}^{s}$ is the estimated, reparameterized satellite clock error of L1/L5 and P1/P5 and the $\delta_{1,2}^{s}$ is the estimated, reparameterized satellite clock error of L1/L2 and P1/P2. The combined UPDs of Equation (6), named phase based IFCB, is computed using the differenced ionosphere-free phase combination of Equation (5). Its time-varying characteristic is analyzed in Montenbruck et al. [14]. The combined hardware delay biases of code in Equation (6), is estimated as a constant for each day in $\mathrm{Li}$ et al. [28]. It is clear that the current studies neglect the time-varying characteristic of the bias in the code observations. This is bad for overall understanding of the code observations, their biases and their impact on the related application, for example positioning.

\section{Data and Experiments}

The twelve-month (1 January-31 December 2016) triple-frequency GPS data set from 114 IGS stations is processed to analyze the time-varying characteristic of the code bias and validate the presented approach. The distribution of the 114 IGS stations is shown in Figure 1. Data is sampled at $30 \mathrm{~s}$ and cut-off elevation is set to 10 degrees. According to the presented method for estimating the combined code bias, the twelve-month single-day combined code biases are estimated. The single-day constant part of the combined code bias of $b_{1,2,5}^{s}$ is computed with the IGS DCB products of DCB (P1-P2) and DCB (P1-P5) based on Equation (6), while it is computed with the mean of the estimated 24-h series using Equation (8). One-year single-day $b_{1,2,5}^{s}$ values estimated with the presented method for each satellite are analyzed using an FFT (fast Fourier transformation). Based on the observed periods, the 24-h arc $b_{1,2,5}^{s}$ is fitted and modeled:

$$
b_{1,2,5}^{s}(t)=a+e \cdot t+y_{i} \sum_{i=1}^{4} \sin \left(\frac{2 \pi}{T_{i}} \cdot t+\theta_{i}\right)(t=0-24 h)
$$

where $a$ is a constant; $e$ is the coefficient of linear term; $i$ is the order of the harmonics; $T_{i}$ is the period; $\theta_{i}$ is initial phase offset, and $y_{i}$ is the amplitude. 


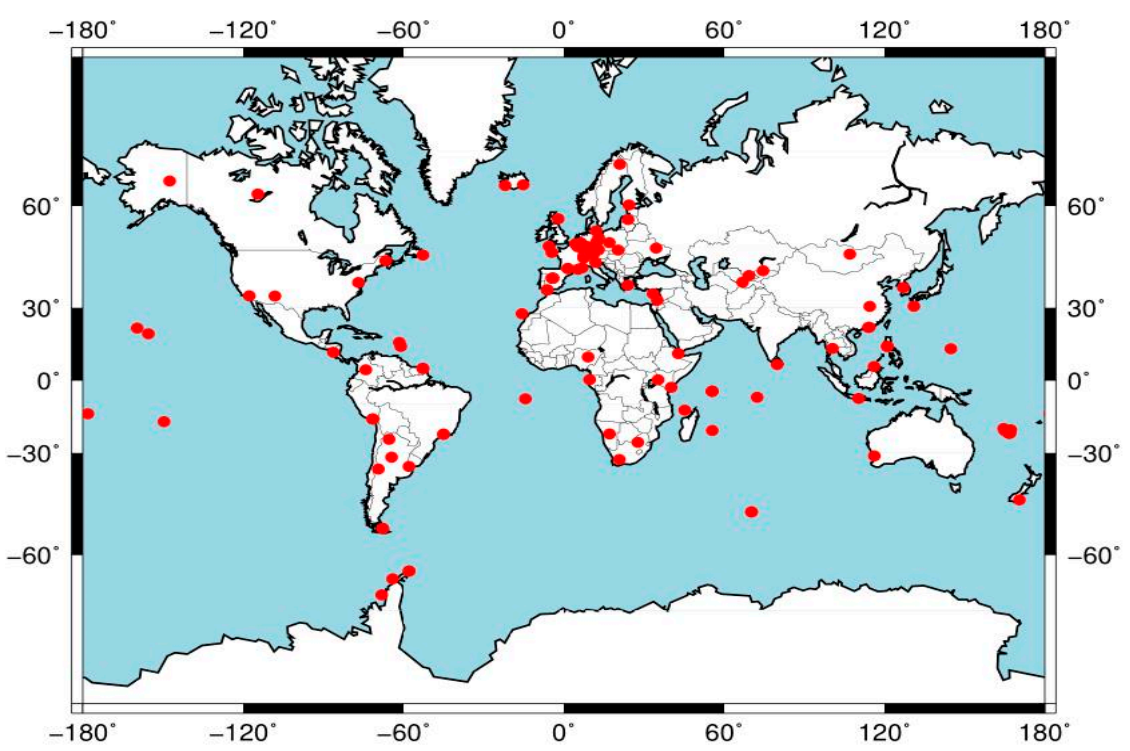

Figure 1. Distribution of the 114 International GNSS Service (IGS) stations.

The satellite clock error is one of the main errors which affect the positioning accuracy. It is important to compute the precise satellite clock errors of code and phase observations for improving the positioning accuracy. The estimated, reparameterized satellite clock errors of L1/L5 and P1/P5 are computed with Equation (10). Therefore, performance of the different results for IFCB estimated with different methods in SPP is used to validate the application of the time-varying code bias in positioning. Methods 1, 2 and 3 are used to compute the IFCB. In Method 1, IFCB is computed using the sum of the combined DCB products and the time-vary part of IFCB estimated with phase observation. In Method 2, IFCB is computed using the sum of the constant part of the combined code bias and the time-vary part of IFCB estimated with phase observation. In Method 3, IFCB is the combined code bias estimating with Section "Estimation of the combined code bias". Five days, 9-13 December 2016, of triple-frequency GPS data from IGS stations of BRUX is processed to analyze the performances of these biases in SPP. The number of the visible BLOCK IIF satellites is illustrated in Figure 2. The skyplot of visible BLOCK IIF satellites observed of BRUX on 9 December is shown in Figure 3. Figures 2 and 3 show that the simultaneous visible BLOCK IIF satellites for many epochs are more than 4 and can ensure that the P1/P5 based SPP is implemented. In SPP processing, the final IGS products of the satellite clock and orbit are used. The elevation-dependent function of Equation (9) is used. The settings for the SPP processing are shown in Table 1. It is very important for real-time processing that the high-accuracy bias is obtained by prediction. Therefore, the prediction performance of the combined code bias is discussed based on 20-day $b_{1,2,5}^{s}$.

Table 1. Settings for the Single point positioning (SPP) processing.

\begin{tabular}{|c|c|c|}
\hline & Model & Settings \\
\hline \multirow{3}{*}{ Measurements } & Ionosphere-free code combination & \\
\hline & Adjustment & Least square \\
\hline & Weighting & Elevation-dependent function \\
\hline \multirow{4}{*}{ Corrections } & DCB (P1-C1) & Products provided by CODE \\
\hline & Tides corrections & Solid tide and Ocean tide correction \\
\hline & PCV & Absolute IGS 08 correction mode \\
\hline & Relativity & Corrected \\
\hline \multirow{3}{*}{ Parameters } & Station coordinates & Estimated \\
\hline & Troposphere & Correction \\
\hline & Receiver clock error & Solved for at each epoch as white noise \\
\hline
\end{tabular}




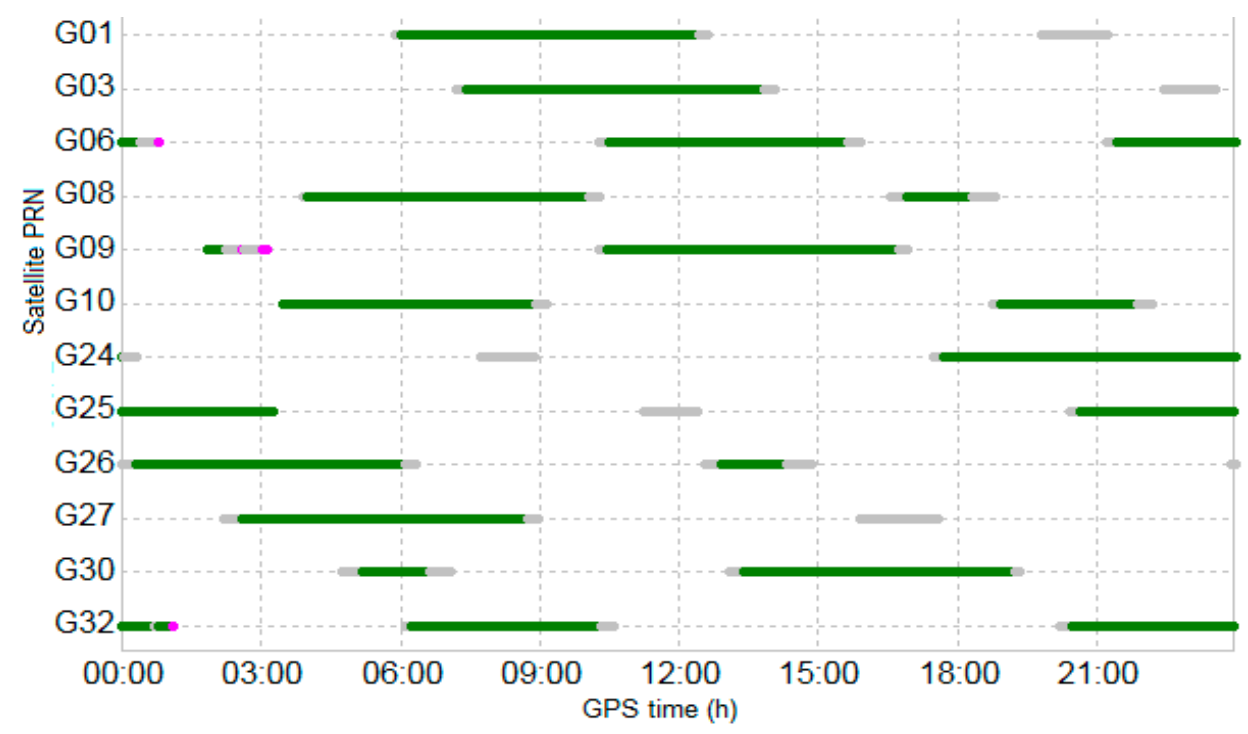

Figure 2. The visible BLOCK IIF satellites, 9 December 2016.

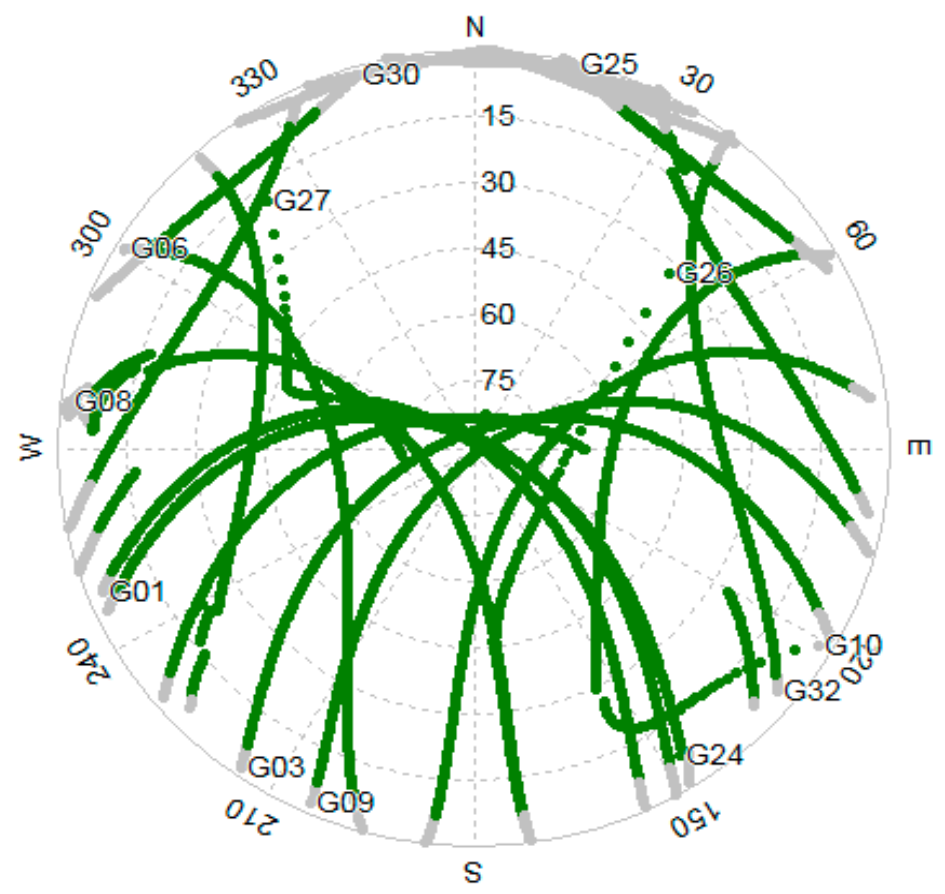

Figure 3. The skyplot of visible BLOCK IIF satellites, 9 December 2016.

\section{Discussion}

In this section, the characteristic of the estimated combined code bias is discussed based on the observed periods and the used model. The constant part of the combined code bias computed with two methods is compared to analyze the difference between the IGS DCB (P1-P2) and DCB (P1-P5) products and estimated combined code bias. The performance of SPP is discussed to validate the application of the estimated combined code bias.

\subsection{Combined Code Biases of $b_{1,2,5}^{s}$}

Analysis of FFT shows that the single-day $b_{1,2,5}^{s}$ has notable variations with the periods of $12,8,6$, 4, 4.8 and $2.67 \mathrm{~h}$. One-year single-day periods $\left(T_{i}(i=1 \sim 4)\right)$ of PRN01, PRN06, PRN26 and PRN30 are illustrated in Figure 4 . The results in Figure 4 indicate that the estimated combined code biases for 
all satellites have stable periods of $12,8,6 \mathrm{~h}$ and the fourth period is irregular. This can be explained that the GPS satellite clock or signals may be affected by other space factors and these factors vary with time. Unfortunately, it is difficult to gain further information about the factors which caused instability of the periods. One day (30 December 2016) estimated results of $b_{1,2,5}^{s}$ for PRN01, PRN06, PRN26 and PRN32 are illustrated in Figure 5, which shows that the estimated combined code bias of $b_{1,2,5}^{s}$ is not the smooth curve and not similar to the combined phase bias of UPD $\mathrm{UP}_{1,2,5}^{s}$ This can be explained by the effect of the noise including the multipath of the code observation on the estimated results. From Figure 5, it can be seen that that the $b_{1,2,5}^{s}$ values for each satellite are different and vary with time, trend of which is different. The variation indicates the combined code bias of $b_{1,2,5}^{s}$ has the time-varying characteristic. This characteristic validates that the combined code bias cannot be replaced by the constant products of satellite DCB (P1-P2) and DCB (P1-P5), although it can be expressed by the function of these DCBs. It also shows that the single-day DCB (P1-P2) and DCB (P1-P5) have the variable characteristic and their time-varying parts are absorbed by the ionosphere delay model in estimating.

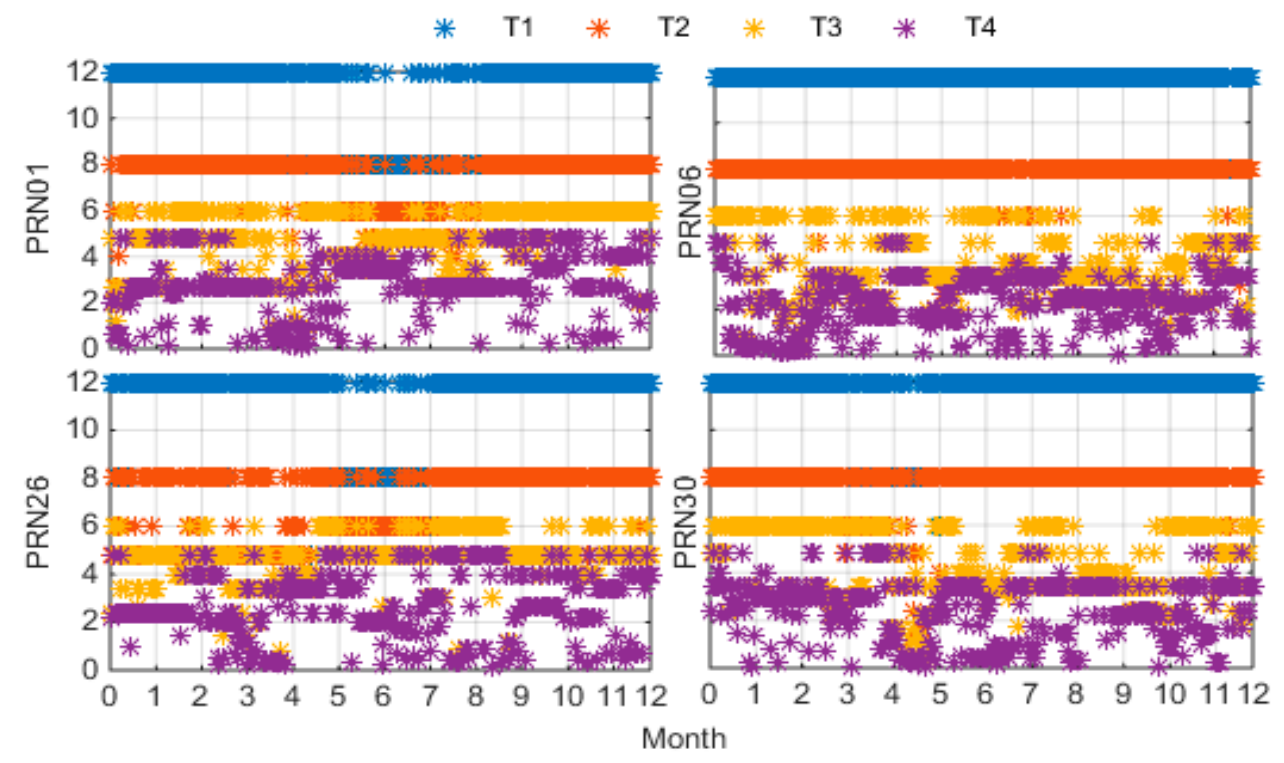

Figure 4. Periods $\left(T_{i}(i=1 \sim 4)\right.$, unit: $\mathrm{h}$ ) of the single-day combined code bias of $b_{1,2,5}^{s}$ for PRN01, PRN06, PRN26 and PRN30 over one year.

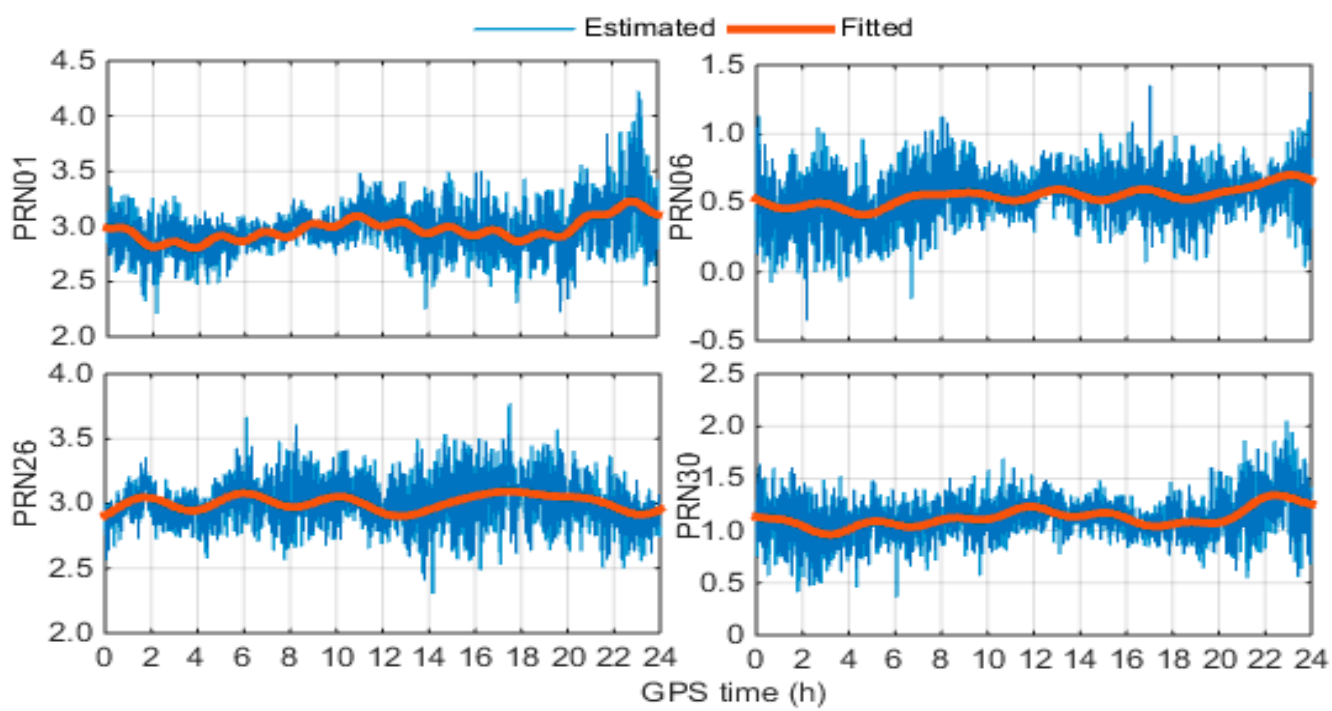

Figure 5. Single-day $b_{1,2,5}^{s}$ (unit: m) series for PRN01, PRN06, PRN26 and PRN30, 30 December 2016. 
The constant part of $b_{1,2,5}^{s}$ computed with two methods of Equations (6) and (8) and their difference is shown in Figure 6. The results in Figure 6 show that the IGS single-day DCB products have the characteristic of the long-term variation. Zhong et al [25] shows that this long-term variation is caused by the GPS satellite replacement. But the results in Figure 6 show that the one-year DCB products have the nonlinear feature except jump and this feature indicates that the factors, which cause the long-term variation, include others, for example the space environment. The difference between the two sets of estimated constant part of $b_{1,2,5}^{s}$ in Figure 6 indicates that the difference between the DCB products and the estimated $b_{1,2,5}^{s}$ using Equation (8) is obvious. This demonstrates that the combined code bias of $b_{1,2,5}^{s}$ can not be replaced by the DCB (P1-P2) and DCB (P1-P5) products.
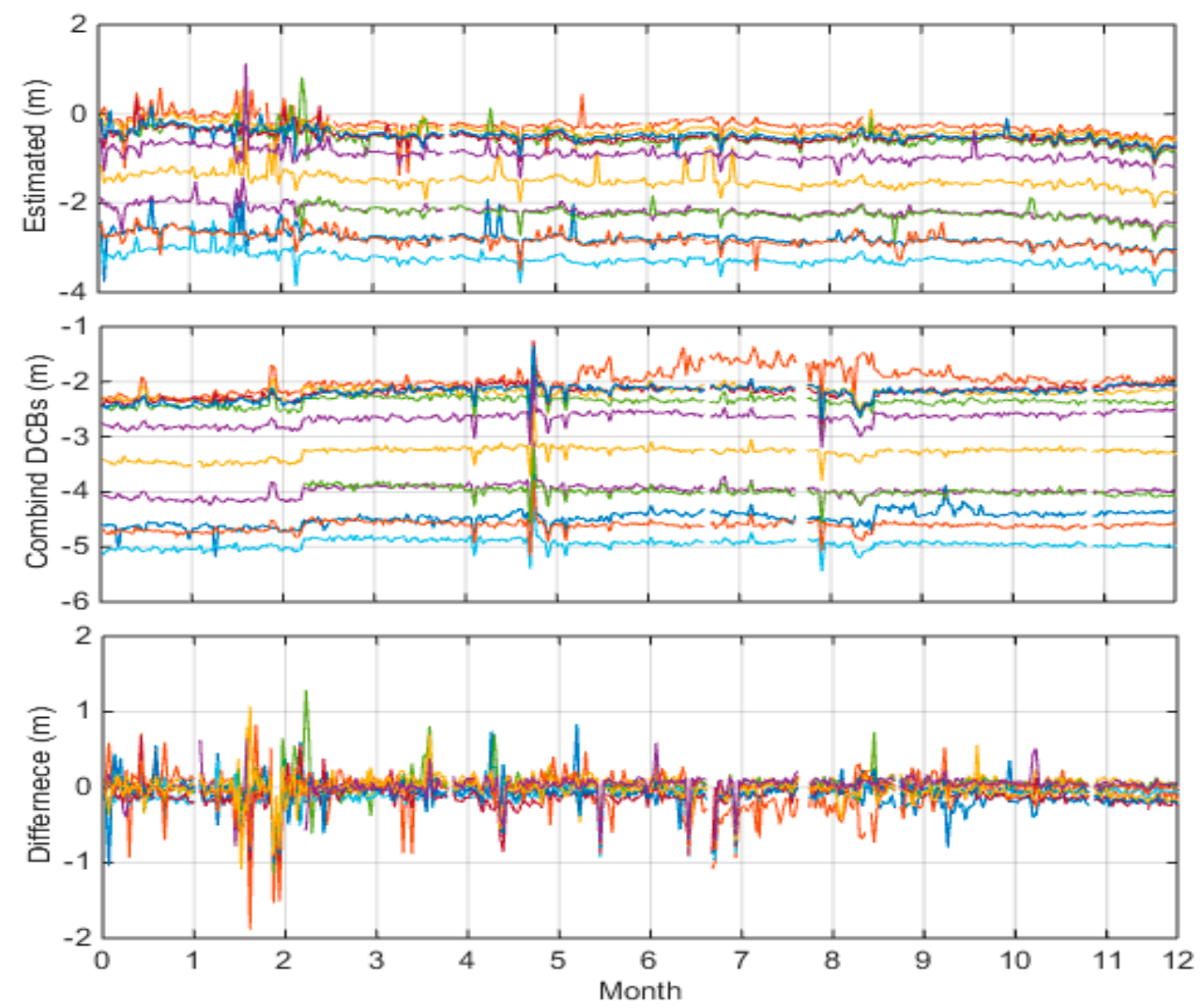

Figure 6. The constant part of $b_{1,2,5}^{s}$ estimated (top) and computed with IGS Differential code bias products (middle) and their difference (bottom).

\subsection{Peformance of SPP}

To evaluate the presented method and validate the impact of the time-varying code bias on the satellite clock error, the performance of the computed satellite clock error with Methods 1, 2 and 3 in the P1/P5 based SPP is analyzed. In processing, the elevation-dependent weight of Equation (9) is used. The corrections such as Earth rotation, Earth tides and relativistic effects are implemented. The tropospheric delay is corrected using the Saastamoinen model. An estimator of least squares is used to solve the epoch-wise coordinates in Methods 1,2 and 3. The IGS final orbit product is used in three strategies. The SPP solution with respect to the ground truth coordinates are computed for three coordinates of north, east and up and the results for the three Methods are illustrated in Figure 7. The RMSs of SPP solution for three coordinates of north, east and up are in Table 2. Figure 7 and Table 2 show that the results for Method of 2 are better than that of 1 . This demonstrates the accuracy of the constant part of the estimated combined code bias is better than that of DCB products and further indicates that the DCB products cannot be used to replace the constant part of IFCB. The results for the Methods of 2 and 3 show that the time-varying part of IFCB estimated with phase observation is better 
than that of code observation. This can be explained by the effect of the noise and multipath on the estimated code-based IFCB.

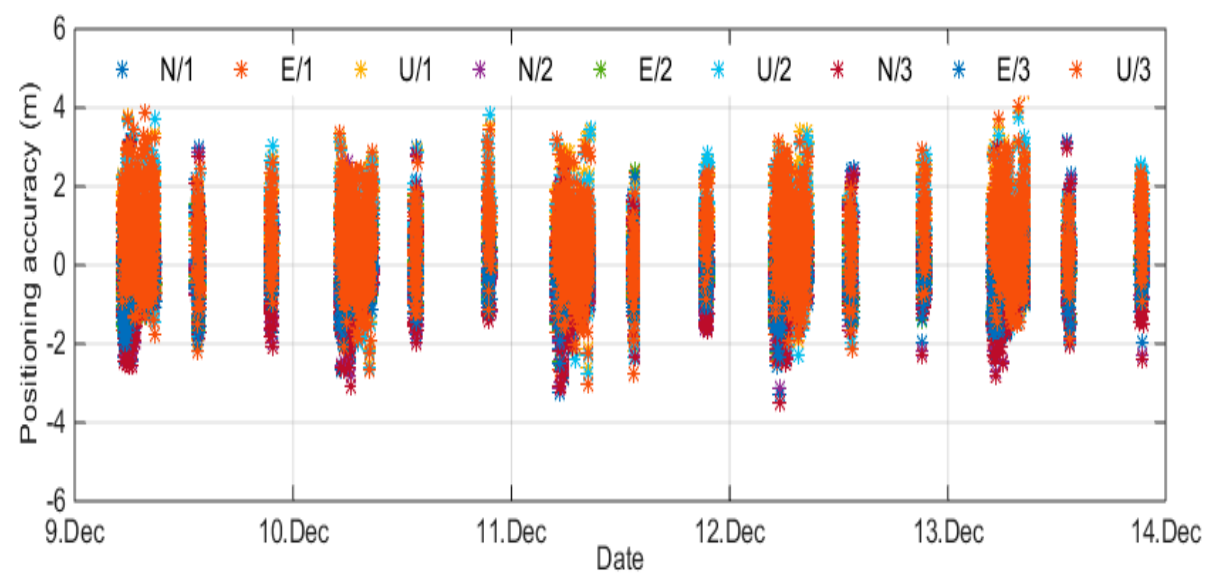

Figure 7. Single point positioning (SPP) solution of Methods 1, 2 and 3 for directions of North (N), East (E) and Up (U).

Table 2. Root mean squares (RMSs) (m) of Single point positioning (SPP) solution for three directions of north, east and up.

\begin{tabular}{cccccccccc}
\hline \multirow{2}{*}{ Date } & \multicolumn{3}{c}{ Method of 1 } & \multicolumn{3}{c}{ Method of 2 } & \multicolumn{3}{c}{ Method of 3 } \\
\cline { 2 - 10 } & North & East & Up & North & East & Up & North & East & Up \\
\hline 9 December & 0.80 & 0.54 & 1.21 & 0.79 & 0.53 & 1.20 & 0.82 & 0.56 & 1.26 \\
\hline 10 December & 0.79 & 0.55 & 1.18 & 0.77 & 0.54 & 1.17 & 0.79 & 0.54 & 1.16 \\
\hline 11 December & 0.82 & 0.53 & 1.09 & 0.78 & 0.51 & 1.04 & 0.80 & 0.53 & 1.05 \\
\hline 12 December & 0.83 & 0.59 & 1.23 & 0.80 & 0.55 & 1.18 & 0.82 & 0.56 & 1.18 \\
\hline 13 December & 0.84 & 0.55 & 1.25 & 0.80 & 0.54 & 1.24 & 0.84 & 0.54 & 1.22 \\
\hline Mean & 0.82 & 0.55 & 1.19 & 0.79 & 0.53 & 1.17 & 0.81 & 0.55 & 1.17 \\
\hline
\end{tabular}

\subsection{Prediction Performance}

The single-day (9 December 2016) estimated $b_{1,2,5}^{s}$ of 12 satellites are used to extrapolate the values of the next days (10-28 December 2016). The mean of the single-day $b_{1,2,5}^{s}$ of 9 December 2016 is used to extrapolate the constant of the next days (10-28 December 2016) and its time-varying part is used to fit the modeling coefficients of each satellite and the fitted coefficients are used to extrapolate the values of the next days (10-28 December 2016). The correction rate is defined as:

$$
c r=\frac{R M S_{E}-R M S_{D}}{R M S_{E}} \times 100 \%
$$

where $R M S_{D}$ is the RMS for the differences between the predicted and estimated values and the $R M S_{E}$ is the RMS of the estimated values. The means of 19-day single-day correction rates of the constant part for each satellite are shown in Figure 8 and the results for the time-varying part are shown in Figure 9 . Figure 8 indicates that the predicted constant part of $b_{1,2,5}^{s}$ reaches $98 \%$ and the attenuation of the predicted accuracy is much less evident. But the Figure 9 shows that the accuracy of the predicted time-varying part decreases significantly with the predicted time. 


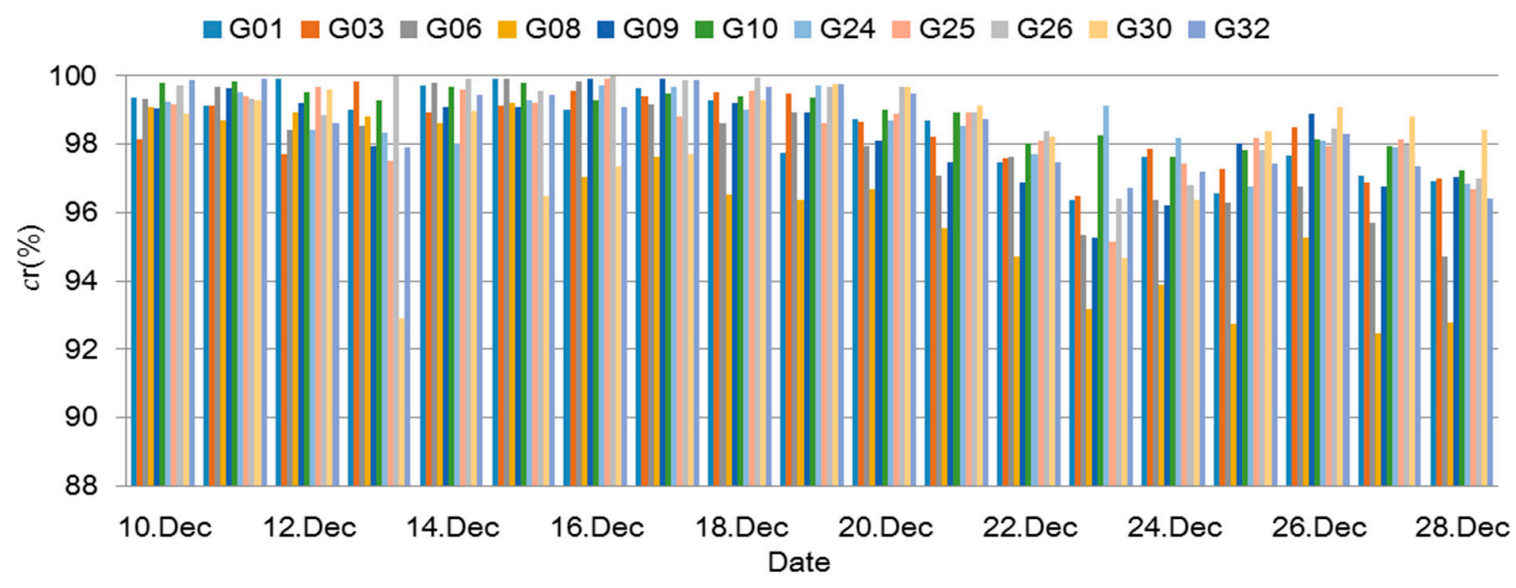

Figure 8. Correction rate $(\%)$ for the constant part.

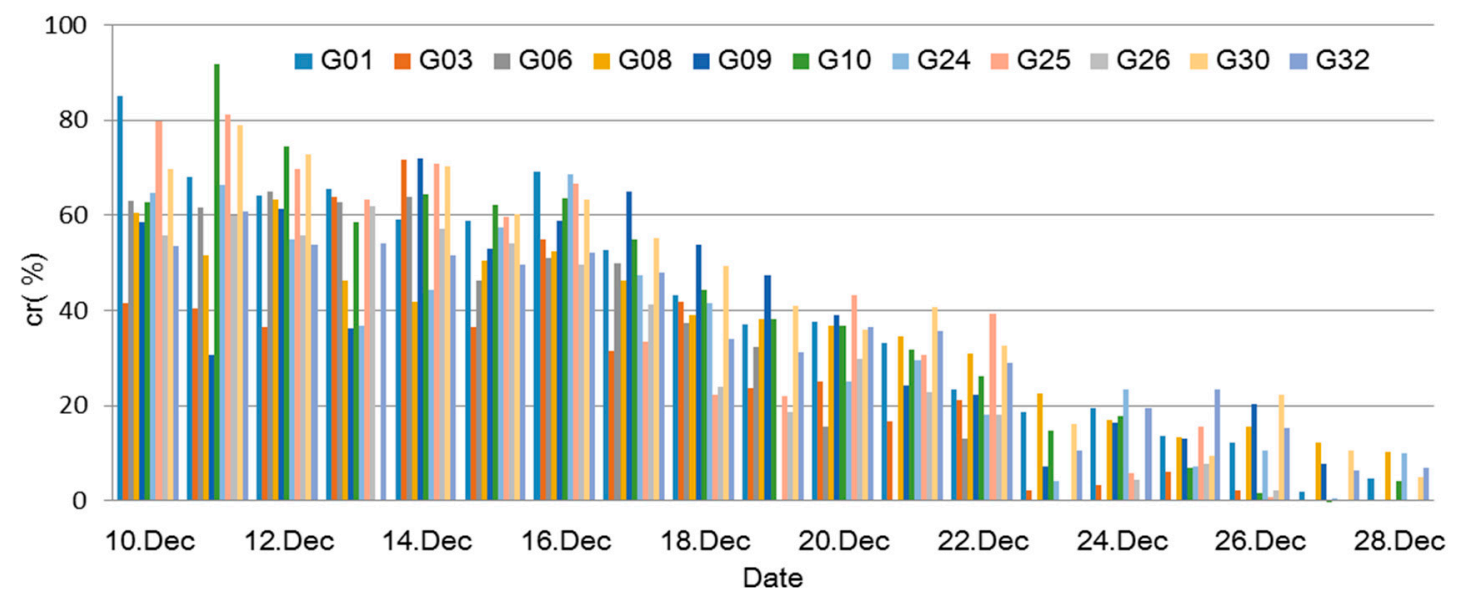

Figure 9. Correction rate (\%) for the time-varying part.

\section{Conclusions}

The characteristics of the biases in the triple-frequency observations are discussed. Meanwhile, a method for estimating the combined code bias is presented. The twelve-month (1 January-31 December 2016) triple-frequency GPS data set from 114 IGS stations is processed to analyze the time-varying characteristic of the code bias using the estimated combined code bias. The time-varying characteristic of the combined code bias is analyzed based on FFT, and one-year results show that the main periods of the combined code bias are $12,8,6,4,4.8$ and $2.67 \mathrm{~h}$. The time-varying characteristic of the combined code bias, which is the combination of DCB (P1-P5) and DCB (P1-P2), shows that the real satellite DCBs are also time-varying. The constant parts of the combined code bias are computed with the IGS DCD products of DCB (P1-P2) and DCB (P1-P2) and the mean of the estimated 24-h combined code bias series and the difference between the two sets of results is obvious. This demonstrate that the combined code bias of $b_{1,2,5}^{s}$ cannot be replaced by the DCB (P1-P2) and DCB (P1-P5) products. The time-varying part of IFCB can be estimated by the phase and code observations and the phase based IFCB is the combinations of the triple-frequency satellite UPDs and code-based IFCB is the function of the DCBs. The performances of the computed the IFCB with different methods in SPP show that the accuracy of the constant part for the estimated combined code bias is better than that of DCB products and further indicates that the DCB products cannot be used to replace the constant part of IFCB. The performance also shows that the time-varying part of IFCB estimated with phase observation is better than that of code observation. This can be explained by the effect of the noise and multipath on the estimated code-based IFCB. The prediction performance shows that the predicted constant part of $b_{1,2,5}^{s}$ can reach $98 \%$ and the attenuation of the predicted accuracy is much less evident. However, the accuracy of the predicted time-varying part decreases significantly with the predicted time. 
Author Contributions: H.L., J.X. and W.Z. conceived and designed the experiments; J.X. and W.Z. performed the experiments and analyzed the data; H.L. wrote the paper; All authors read and approved the final manuscript.

Funding: This research was funded by the National Natural Science Foundation of China (NNSFC), Grant Number 41674029 and the Fundamental Research Funds for the Central Universities, Grant Number 0250219113.

Acknowledgments: The authors would like to thank the International GNSS Service (IGS) communities for providing the products.

Conflicts of Interest: The authors declare no conflict of interest.

$\begin{array}{ll}\text { Abbreviations } \\ \text { GNSS } & \text { Global navigation satellite system } \\ \text { IFCB } & \text { Inter-frequency clock bias } \\ \text { DCB } & \text { Differential code bias } \\ \text { IGS } & \text { International GNSS Service } \\ \text { PPP } & \text { precise point positioning } \\ \text { RMS } & \text { Root Mean Square } \\ \text { UPD } & \text { Uncalibrated phase delays } \\ \text { IFB } & \text { Inter-frequency biases } \\ \text { WL } & \text { wide lane } \\ \text { NL } & \text { narrow lane }\end{array}$

\section{References}

1. Hakansson, M.; Jensen, A.; Horemu, M.; Hedlin, G. Review of code and phase biases in multi-GNSS positioning. GPS Solut. 2017, 21, 849-860. [CrossRef]

2. Sardon, E.; Ruis, A.; Zarraoa, N. Estimation of the transmitter and receiver differential biases and the ionospheric total electron content from Global Positioning System observations. Radio Sci. 1994, 29, 577-586. [CrossRef]

3. Goodwin, G.; Breed, A. Total electron content in Australia corrected for receiver/satellite bias and compared with IRI and PIM predictions. Adv. Space Res. 2001, 27, 49-60. [CrossRef]

4. Otsuka, Y.; Ogawa, T.; Saito, A.; Tsugawa, T.; Fukao, S.; Miyazaki, S. A new technique for mapping of total electron content using GPS network in Japan. Earth Planets Space 2002, 54, 63-70. [CrossRef]

5. Klobuchar, J.A. Ionospheric time-delay algorithm for single frequency GPS users. IEEE Trans. Aerosp. Electron. Syst. 1987, 23, 325-331. [CrossRef]

6. Schaer, S. Mapping and Predicting the Earth's Ionosphere Using the Global Positioning System. Ph.D. Thesis, Astronomical Institute, University of Bern, Bern, Switzerland, 1999.

7. Tu, R.; Ge, M.; Zhang, H.; Huang, G. The realization and convergence analysis of combined PPP based on raw observation. Adv. Space Res. 2013, 52, 211-221. [CrossRef]

8. Dow, J.; Neilan, R.; Rizos, C. The International GNSS service in a changing landscape of Global Navigation Satellite Systems. J. Geod. 2009, 83, 191-198. [CrossRef]

9. Gao, Y.; Lahaye, F.; Liao, X.; Heroux, P.; Beck, N.; Olynik, M. Modeling and estimation of C1-P1 bias in GPS receivers. J. Geod. 2001, 74, 621-626. [CrossRef]

10. Tian, Y.; Ge, M.; Neitze, F.; Zhu, J. Particle filter-based estimation of inter-system phase bias for real-time integer ambiguity resolution. GPS Solut. 2017, 21,949-961. [CrossRef]

11. Ge, M.; Gendt, G.; Rothacher, M.; Shi, C.; Liu, J. Resolution of GPS carrier-phase ambiguities in precise point positioning (PPP) with daily observations. J. Geod. 2008, 82, 389-399. [CrossRef]

12. Wanninger, L. Carrier-phase inter-frequency biases of GLONASS receivers. J. Geod. 2011, 86, $139-148$. [CrossRef]

13. Zhang, B.; Teunissen, P.; Yuan, Y. On the short-term temporal variations of GNSS receiver differential phase biases. J. Geod. 2017, 91, 563-572. [CrossRef]

14. Montenbruck, O.; Hugentobler, U.; Dach, R.; Steigenberger, P.; Hauschild, A. Apparent clock variations of the Block IIF-1 (SVN62) GPS satellite. GPS Solut. 2012, 16, 303-313. [CrossRef]

15. Li, H.; Zhou, X.; Wu, B.; Wang, J. Estimation of the inter-frequency clock bias for the satellites of PRN25 and PRN01. Sci. China-Phys. Mech. Astron. 2012, 55, 2186-2193. [CrossRef] 
16. Li, H.; Zhou, X.; Wu, B. Fast estimation and analysis of the inter-frequency clock bias for Block IIF satellites. GPS Solut. 2013, 17, 347-355. [CrossRef]

17. Leick, A.; Rapoport, L.; Tatarnikov, D. GPS Satellite Surveying, 4th ed.; Wiley: New York, NY, USA, 2015.

18. Li, H.; Xu, T.; Li, B.; Huang, S.; Wang, J. A new differential code bias (C1-P1) estimation method and its performance evaluation. GPS Solut. 2016, 20, 321-329. [CrossRef]

19. Li, H.; Li, B.; Lou, L.; Yang, L.; Wang, J. Impact of GPS differential code bias in dual- and triple-frequency positioning and satellite clock estimation. GPS Solut. 2017, 21, 897-903. [CrossRef]

20. Levine, J. A review of time and frequency transfer methods. Metrologia 2008, 45, 162-174. [CrossRef]

21. Montenbruck, O.; Hauschild, A.; Steigenberger, P. Differential Code Bias Estimation using Multi-GNSSO bservations and Global Ionosphere Maps. Navigation 2014, 61, 191-201. [CrossRef]

22. Li, Z.; Yuan, Y.; Li, H.; Ou, J.; Huo, X. Two-step method for the determination of the differential code biases of COMPASS satellites. J. Geod. 2012, 86, 1059-1076. [CrossRef]

23. Wang, N.; Yuan, Y.; Li, Z.; Montenbruck, O.; Tan, B. Determination of differential code biases with multi-GNSS observations. J. Geod. 2016, 90, 209-228. [CrossRef]

24. Herna'ndez-Pajares, M.; Juan, J.; Sanz, J.; Orus, R.; Garcia-Rigo, A.; Feltens, J.; Komjathy, A.; Schaer, C.; Krankowski, A. The IGS VTEC maps: A reliable source of ionospheric information since 1998. J. Geod. 2009, 83, 263-275.

25. Zhong, J.; Lei, J.; Dou, X.; Yue, X. Is the long-term variation of the estimated GPS differential code biases associated with ionospheric variability? GPS Solut. 2016, 20, 313-319. [CrossRef]

26. Choi, B.; Lee, S. The influence of grounding on GPS receiver differential code biases. Adv. Space Res. 2018, 62, 457-463. [CrossRef]

27. Yasyukevich, Y.; Mylnikova, A.; Kunitsyn, V.; Padokhin, A. Influence of GPS/GLONASS differential code biases on the determination accuracy of the absolute total electron content in the ionosphere. Geomagn. Aeron. 2015, 55, 763-769. [CrossRef]

28. Li, H.; Li, B.; Xiao, G.; Wang, J.; Xu, T. Improved method for estimating the inter-frequency satellite clock bias of triple-frequency GPS. GPS Solut. 2016, 20, 751-760. [CrossRef]

(C) 2019 by the authors. Licensee MDPI, Basel, Switzerland. This article is an open access article distributed under the terms and conditions of the Creative Commons Attribution (CC BY) license (http:/ / creativecommons.org/licenses/by/4.0/). 